\title{
Investigation of Ti64 sheathed cellular anatomical structure as a Tibia implant
}

\author{
Aaron Vance ${ }^{1}$, Klaudio Bari ${ }^{1}$ and Arun Arjunan ${ }^{1}$ \\ ${ }^{1}$ School of Engineering, Faculty of Science and Engineering, University of Wolverhampton \\ Telford Innovation Campus, TF2 9NT, United Kingdom \\ E-mail: $\underline{\text { A.Vance@wlv.ac.uk }}$ \\ Received xxxxxx \\ Accepted for publication $\mathrm{xxxxxx}$ \\ Published xxxxxx
}

\begin{abstract}
In order to reduce stress shielding following a segmental bone replacement surgery requires stiffness matching strategies between the host bone and the implant are required. Carefully engineered implant geometry that can mimic the mechanical performance of the host bone is required to achieve this. The development of Additive Layer Manufacturing (ALM) techniques such as Direct Metal Laser Sintering (DMLS) allows for the fabrication of complex geometries that can achieve targeted mechanical performance. Consequently, this work introduces a sheathed Ti6Al4V additively manufactured tibial implant that mimics the circumferential anatomy of the host bone. Performance evaluation of the implant was carried using experimental and numerical technique under axial compression. Furthermore, the influence of sheathing strategy and sheath thickness on the compressive performance of the implant is parametrically analysed. The results of this study shows a promising sheathed implant that can replace a defective tibia bone segment. The implant is superior to conventional porous implants as it allows for easy implantation in surgical operation and allows for the reduction of stress shielding.
\end{abstract}

Keywords: Sheathed Tibia Implant; Stress Shielding, Direct Metal Laser Sintering, Finite Element Analysis, Anatomical Design, Compression, Lattice Structure

\section{Introduction}

Additive Layer Manufacturing enables tailored porous material to be designed with desirable mechanical properties. Lattice architecture, specifically periodic open cellular unit cells are of interest. Previous studies have shown lattice structure architecture can affect both mechanical, biocompatibility and bone reintegration $(1,2)$. However, Implants for bone reintegration should meet certain criteria to, including mechanical properties comparable that of the host bone, biocompatibility and biodegradability at a rate commensurate with remodelling $(3,4)$. Implants serve primarily as osteoconductive moieties, since new bone is deposited by creeping substitution from adjacent living tissue (5).

The biocompatibility of commercially used titanium alloy (Ti6Al4V) is well founded in medical environments $(6,7)$. However, the use of traditional Ti6Al4V implants in-vivo presents mechanical property mismatch, specifically the phenomenon of stress shielding (8).

Historically orthopaedic implants have been manufactured using cast or forged solid pieces of metal, that are order of magnitude stiffer than natural bone. Implants should have matching stiffness and strength properties of hard tissue $(9,10)$. Computational tomography (CT) tools and the generation of anatomical models are enabling improved 
patient care by providing insight into biological responses resulting from patient trauma.

CT technologies are well founded within medical and engineering sectors (11), convergence of disciplines is enabling the generation of three-dimensional bio-models, $(9,10,12)$. Additive layer manufacturing (ALM) methods have enabled production of anatomical products derived from bespoke bio-models. Complex and additively manufactured biomaterials have been shown to improve bone regeneration and osseointegration (13-15).

In this paper both CT and ALM (16) are combined to generate a bespoke bio-model of the tibia bone segment. Using the bio-model as reference, linearly multiplied unit cells were designed to obtain the anatomical profile. Numerical analysis was performed to evaluate the suitability of the anatomical tibia implant under compression. Under identical boundary conditions, mechanical testing was performed to validate the finite element predictions (17-19). Furthermore, parametric analysis on the influence of sheath strategy and thickness on the mechanical performance of the implant evaluated. In addition, the surface roughness of the Ti6Al4V implant was characterised using optical microscopy to characterise the potential for bone growth.

\section{Methodology}

\subsection{Bio-model of the segmented bone}

From the tibia bone shown in Figure 1, an arbitrary section of length $21.2 \mathrm{~mm}$ was cut using a rotary saw blade. Using the segmented bone, the Bruker Skyscan 2211 CT scanner was used to generate the segmental bone topography. Even though the process created a fine resolution 3D model, for computational efficiency only the surface topography was extracted in the form of Standard Tessellation Language (STL).

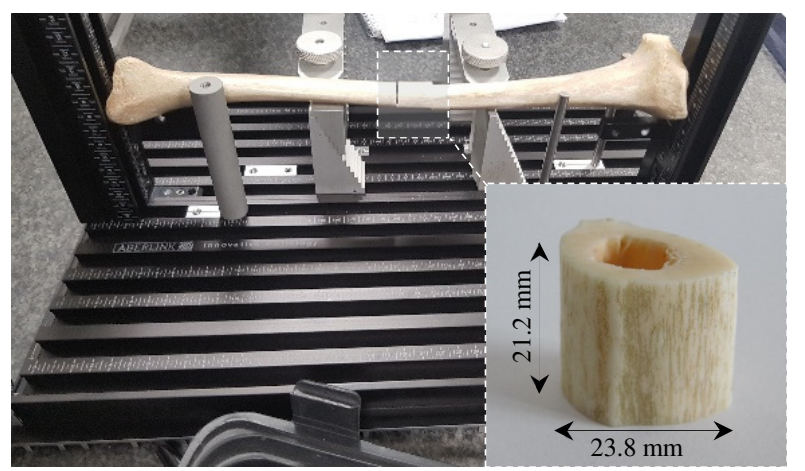

Figure 1. Segmented tibial section considered for the generation of the digital bio-model.

The STL file was then imported into a commercial Computer Aided Design (CAD) package SolidWorks and a digital model was generated using Bézier splines. The resulting model as shown in Figure 2 was used to generate the volumetric anatomical bio-model. A bottom up modelling approach was then used to generate a singular unit cell to fill the anatomical volume.

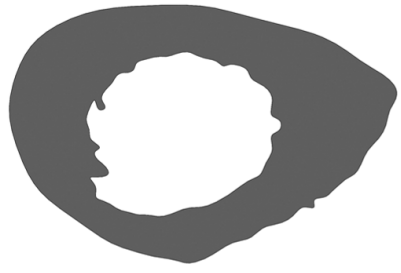

Figure 2. Topology of the digital bio-model mimicking the circumferential anatomy of the host bone.

Numerous unit cell designs were investigated both in terms of the relative densities and stress distribution (20). Based on the results obtained the most suitable unit cell with dimensions shown in Figure 3 was considered for the implant design. A uniform scaling factor $\left(s_{f}\right)$ of 0.424 was used to fit five-unit cell within the segmented height of the bio-model.
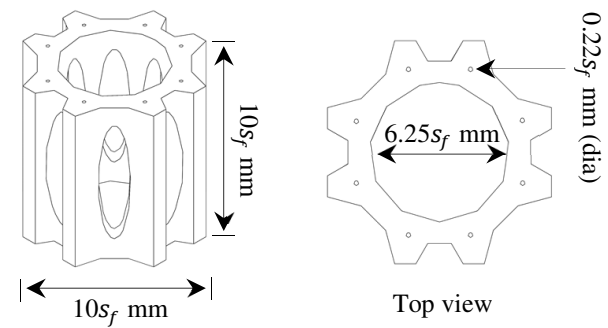

Figure 3. Final unit cell design considered for the implant.

The generated bio-model was filled with the selected unit cell using triaxle linear patterning. After linear patterning, the Boolean combine and difference operation was used to produce the three-dimensional anatomical lattice structures. A total of four implant categories were considered for the anatomical design as shown in Figure 4. The selection was based on whether the design featured a sheath including its location (inside, outside or both). Based on the sheath thickness, there are slight differences in the anatomical profile due to geometrical limitations.

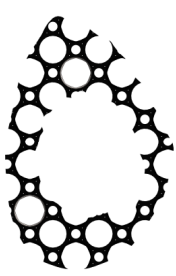

NS

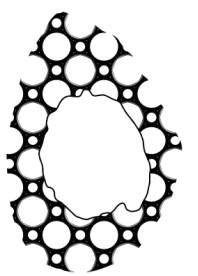

IS

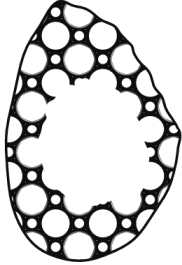

OS

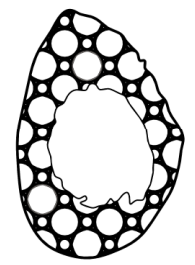

IOS
Figure 4. Four design categories considered for the anatomical implant. 
The parametric variables considered for the four design categories are listed in Table 1. Design NS feature no sheath, while IS and OS features inside and outside sheath respectively. Whereas, Design IOS features both inside and outside sheath.

Table 1. Parameters associated with the four implant design cases considered.

\begin{tabular}{lccc}
\hline \multicolumn{1}{c}{ Design category } & $\begin{array}{c}\text { Implant } \\
\text { Design }\end{array}$ & $\begin{array}{c}\text { Inner } \\
\text { sheath } \\
\text { thickness } \\
\text { (mm) }\end{array}$ & $\begin{array}{c}\text { Outer } \\
\text { sheath } \\
\text { thickness } \\
\text { (mm) }\end{array}$ \\
\hline $\begin{array}{l}\text { No Sheath } \\
\text { (NS) }\end{array}$ & NS1 & 0 & 0 \\
\hline Inner Sheath & IS1 & 0.1 & 0 \\
(IS) & IS2 & 0.2 & 0 \\
\hline & IS3 & 0.3 & 0 \\
Outer Sheath & OS1 & 0 & 0.2 \\
(OS) & OS2 & 0 & 0.3 \\
& OS3 & 0 & 0.4 \\
\hline Inner and Outer sheath & OS4 & 0 & 0.5 \\
(IOS) & OS5 & 0 & 0.6 \\
\hline
\end{tabular}

\subsection{Numerical analysis}

Three-dimensional (3D) numerical analysis was carried out using the Finite Element Method (FEM). A commercial FE code as part of the SolidWorks 2018 simulation suite was used to analyse all the design variations as listed in Table 1.

A solid elemental matrix that featured a linear tetrahedral element defined by four corner nodes connected by six straight edges was used for the mesh. Using a mesh sensitivity analysis, further mesh refinements were carried out until the results converged. The resulted elemental and nodal distribution for the converged finite element model was 4471889 and 6844490 respective. The elemental matrix was solved using an Intel Core i7-6700 CPU at $3.40 \mathrm{GHz}$ with a maximum RAM allocation of $128 \mathrm{~GB}$. The resultant mesh and solution time were approximately 18 and 21 minutes respectively.

The boundary conditions were applied to replicate the physical test conditions as shown in Figure 5. The bottom surface of the implant was fully constrained to zero displacement and a perfectly axial normal surface pressure was applied at the top face. For the implant, the surface pressure applied on the top surface was calculated by dividing $1000 \mathrm{~N}(\sim 100 \mathrm{~kg})$ by the surface area of the biomodel which was $73.5 \mathrm{~mm}^{2}$ resulting in a pressure of 13.6MPa.

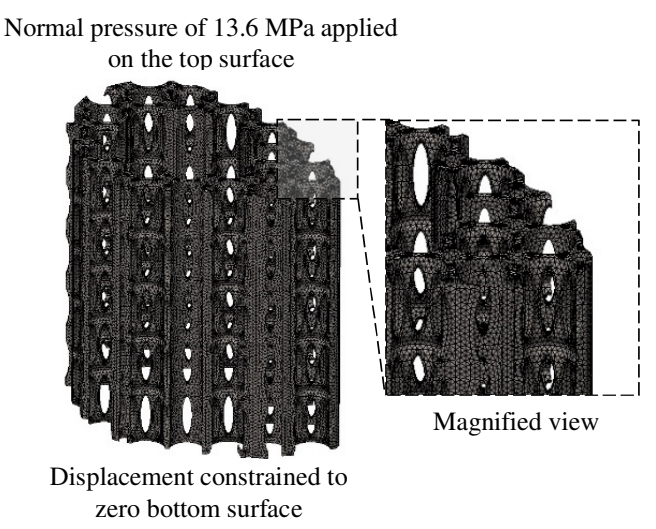

Figure 5. Boundary conditions and mesh resolution used for the numerical simulation.

The implant lattice was modelled as a single continuum body with Ti64 (Ti6Al4V) material properties. An isotropic material model with Young's Modulus $\left(E_{B}\right)$ of $104.8 \mathrm{GPa}$, density $\left(\rho_{B}\right)$ of $4428.78 \mathrm{~kg} / \mathrm{m}^{3}$ and Poisson's ratio of 0.3 were used. From the FEA results, the Eqn. 1 (20) was used to predict the Young's Modulus $\left(E_{F E A}\right)$ of the implant design:

$$
E_{F E A}=\frac{E_{U C}}{E_{B}} \times \frac{\sigma_{y_{F E A}}}{\varepsilon_{y_{F E A}}}
$$

Where, $E_{U C}$ is the relative Modulus of the unit cell used for the implant design, $E_{B}$ is the Young's modulus of the bulk material, $\sigma_{y_{F E A}}$ and $\varepsilon_{y_{F E A}}$ is the maximum stress and strain in y direction. The strain is obtained as the ratio of the maximum axial deflection from FEA $(\mathrm{mm})$ to original height of the implant, which is $21.2 \mathrm{~mm}$.

\subsection{Direct metal laser sintering (DMLS)}

The direct metal laser sintering was performed using Ytterbium fibre laser system that uses Ti64 particles $\approx 25-40$ $\mu \mathrm{m}$ in diameter. Laser scanning speed was $7 \mathrm{~mm} / \mathrm{s}$ with a wavelength of $1054 \mathrm{~nm}$. The laser power was set at a constant 195 watt and spot size of $40 \mu \mathrm{m}$ was used. Only the implant design IOS was manufactured as it was the most complex. The parts were printed using EOS M290 additive manufacturing machine. Build support were generated at $1 \mathrm{~mm}$ and removed using submerged wire Electro-Discharge Machining (EDM). After the printing process, the implant was heat treated at $650^{\circ} \mathrm{C}$ for 3 hours in an argon environment.

\subsection{Experimental testing and surface characterisation}

The compression test was conducted using the ZwickRoell Z1474 testing rig. A maximum load of $90 \mathrm{kN}$ with a force shutdown threshold of $20 \%$ and maximum deformation of $10 \%$ was applied. The threshold and deformation value 
were used to ensure the compression plates did not collide. A stress strain curve was obtained for further analysis.

The surface roughness characterisation was carried out using the Olympus LEXT OLS3100 confocal laser scanning microscope.

\section{Results}

\subsection{Numerical results}

The unit cell used as the basis of the implant design was characterised using the Maxwell criteria, the results of which are presented in Table 1. The density of the unit cell was evaluated using Eqn. 2:

$$
\rho_{U C}=\frac{m_{U C}}{V_{B(U C)}}
$$

Where, the $m_{U C}$ and $V_{B(U C)}$ are the mass of the unit cell and volume of the bulk material respectively. Further to this, relative density of the unit cell to bulk Ti64 was calculated based on Eqn. 3:

$$
\rho_{r(U C)}=\frac{\rho_{U C}}{\rho_{B}}
$$

The relative Young's Modulus $\left(E_{(U C)}\right)$ was then calculated using Eqn. 4:

$$
E_{(U C)} \approx E_{B} \cdot\left(\rho_{r(U C)}\right)^{2}
$$

Table 2. Properties of unit cell.

\begin{tabular}{ll}
\hline Properties & values \\
\hline Unit cell relative density $\left(\rho_{r(U C)}\right)$ & 0.2461 \\
$\mathrm{~b}$ (number of beams) & 24 \\
$\mathrm{j}$ (number of joints) & 16 \\
$M=b-3 j+6$ & -18 \\
Relative Young's Modulus $E_{(U C)}(\mathrm{GPa})$ & 6.35 \\
\hline
\end{tabular}

A total of 10 implant design under four categories as listed in Table 1 were developed based on the same unit cell. Following the Maxwell criterion for lattice structures, the relative density $\left(\rho_{r(i m p)}\right)$ of the implant designs are evaluated as listed in Table 3. The bulk volume of the bone $\left(V_{\text {bone }}\right)$ was derived from digital bio-model as $3816 \mathrm{~mm}^{3}$.

The highest relative density of the implant $\left(\rho_{r(i m p)}\right)$ was exhibited by the design OS5, which featured only the outer sheath having a thickness of $0.6 \mathrm{~mm}$. The lowest $\rho_{r(\mathrm{imp})}$ was exhibited by NS1 which featured no sheath at all. It can be also seen that after rounding, design OS1 and IS3 feature similar relative density. A similar trend can be observed for designs OS2 and IOS1 despite featuring different sheathing strategies. Overall, the relative densities for all designs are within a 0.25 to 0.43 range which relates to a porosity $(\phi)$ range of $57-75 \%$ which is conducive for bone growth.

Table 3. Properties of the implant designs considered.

\begin{tabular}{llllc}
\hline $\begin{array}{c}\text { Implant } \\
\text { Design }\end{array}$ & $\begin{array}{c}\text { Volume } \\
\left(\mathbf{m m}^{\mathbf{3}}\right)\end{array}$ & Mass $(\mathbf{g})$ & $\begin{array}{c}\text { Relative } \\
\text { density } \\
\rho_{r(\mathrm{imp})}\end{array}$ & $\begin{array}{c}\text { Porosity } \\
\mathbf{\%} \\
(\phi)\end{array}$ \\
\hline NS1 & 0949.48 & 4.21 & 0.25 & 75.09 \\
IS1 & 1025.52 & 4.54 & 0.27 & 73.14 \\
IS2 & 1098.66 & 4.87 & 0.29 & 71.18 \\
IS3 & 1172.26 & 5.19 & 0.31 & 69.29 \\
OS1 & 1179.37 & 5.22 & 0.31 & 69.11 \\
OS2 & 1292.62 & 5.72 & 0.34 & 66.15 \\
OS3 & 1405.13 & 6.22 & 0.37 & 63.20 \\
OS4 & 1519.26 & 6.73 & 0.40 & 60.18 \\
OS5 & 1632.78 & 7.23 & 0.43 & 57.22 \\
IOS1 & 01292.9 & 5.73 & 0.34 & 66.27 \\
\hline
\end{tabular}

Figure 6 shows the theoretical Young's modulus ( $E_{\text {theory }}$ ) plotted against the relative density. As expected, a higher relative density gives rise to a higher modulus. The highest Young's modulus was exhibited by OS5 that feature only an outer sheath and lowest by NS1 (no sheath).

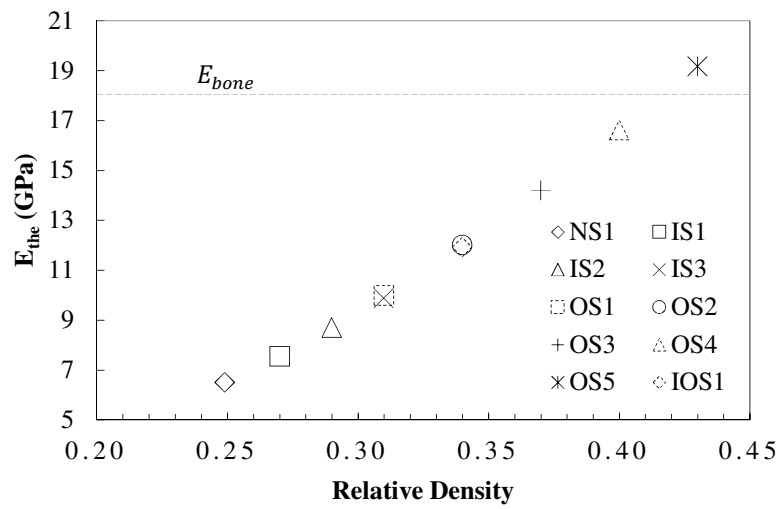

Figure 6. Theoretical Young's modulus predicted for the implant designs considered and their associated relative densities.

The segmented bone was experimentally tested and resulted in a Young's modulus $\left(E_{\text {bone }}\right)$ of $18.01 \mathrm{GPa}$. Comparing the theoretical performance of the implant designs with the performance of the segmented bone, designs OS5 and OS4 shows comparable performance with difference of $+6.29 \%$ and $-8.02 \%$ respectively.

Figure 7 shows the von Mises stress distribution compared against the four design categories under an uniform contour. The theoretical limit of the contour was obtained by dividing a $1000 \mathrm{~N}$ working load with the representative crosssectional area $\left(73.5 \mathrm{~mm}^{2}\right)$ of the bio-model normal to the load. This resulted in a theoretical maximum of $13.7 \mathrm{MPa}$ (rounded). Comparing the results, it is evident that, with or without the sheath, the stress is uniformly distributed within 
the lattices. However, using the external sheath causes a higher stress concentration compared to internal sheath of similar thickness. Consequently, sheath thickness must be carefully chosen to not dominate as the primary load bearing structure. For IOS1, it is evident that the combination of different sheath thickness ensures that the stress is uniformly distributed within the structure. Nevertheless, varying the sheathing strategy can be adopted to prevent maladapted stress concentration within segmented section.

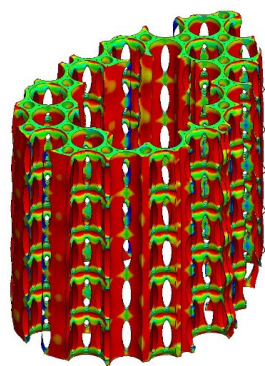

(a)

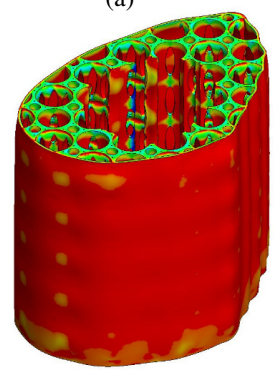

(c)

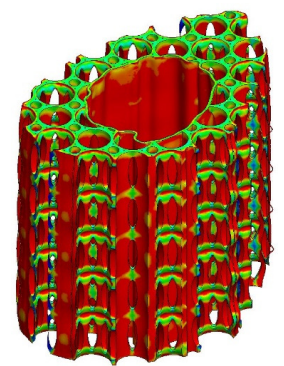

(b)

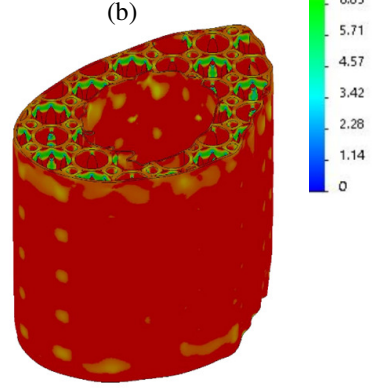

(d)
Figure 7. von Mises stress (MPa) for the four implant categories considered where (a) NS1, (b) IS2 (c) OS1 and (d) IOS1.

The maximum normal stress values obtained for all designs are shown in Figure 8. Comparing the normal stress for the design featuring only inner sheath (IS), it is evident that increasing the sheath thickness induces a higher stress concentration with in the design.

Increasing the inner sheath thickness from 0.1 to 0.2 the increase in stress concentration was $2.76 \%$. However, when the thickness was increased from 0.2 to 0.3 , the stress concentration increased $33 \%$. Accordingly, to enable an even distribution of the stress while going for an IS design strategy, the sheath thickness must be kept to a minimum, preferably $\leq 0.1 \mathrm{~mm}$. However, compared to the sheath-less design, having a smaller inner sheath distributes the stress more evenly. Nevertheless, the Ti64 DMLS process requires a minimum of $100 \mu \mathrm{m}$ for a repeatable print. A $300 \mu \mathrm{m}$ is often considered repeatable within an accuracy of $\pm 1 \%$ for bulk compression.

For the outer sheath design, both increasing and decreasing the thickness seems to affect the stress concentration drastically. However, the stress concentration reduced at thicknesses $0.3,0.4$ and 0.5 in comparison to 0.2 and 0.6. Furthermore, the outer sheath strategy seems to be superior from a stress perspective compared to IS and NS strategy.

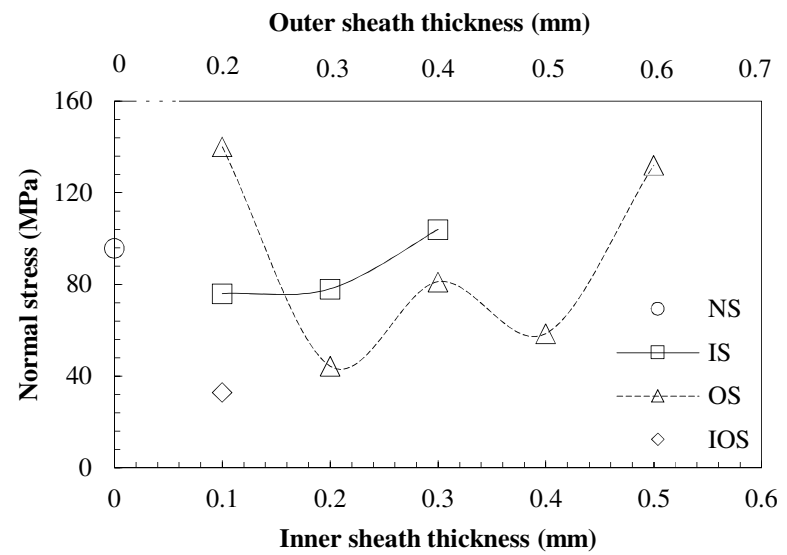

Figure 8. Normal stress for the implant designs obtained from finite element analysis.

Comparing the performance of the non-sheath design with the inner and outer sheath strategy (IOS), it is evident that the performance of IOS is superior to that of NS. Consequently, the stress concentration reduced drastically when both inner and outer sheath was introduced in comparison to a nonsheath design. Therefore, in order to reduce stress concentration within the implant design, the IOS sheath strategy is suitable in comparison to the other alternatives considered. Overall, IOS1 showed the lowest stress concentration, followed by OS2 and OS4.

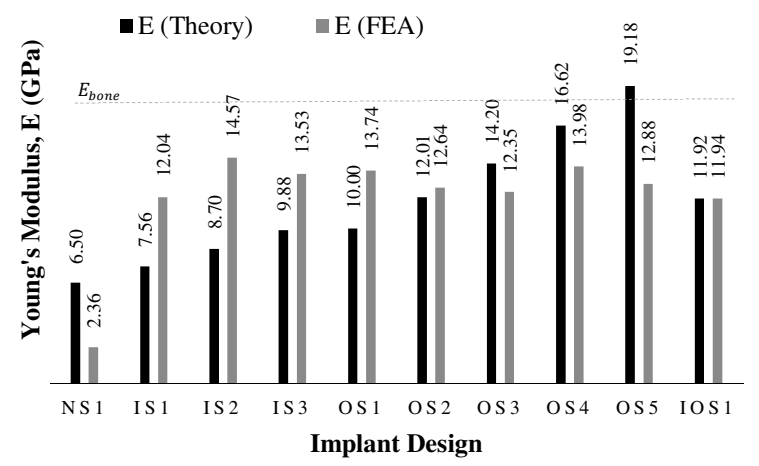

Figure 9. Comparison of Young's modulus from Maxwell criterion and Finite element analysis.

Comparing the theoretical Young's modulus with FEA as shown in Figure 9. It is evident that that Maxwell criteria cannot accurately predict the stiffness of the anatomical lattice geometry that does not feature a sheath as the stress concentration is dominant. This is due to the irregular anatomical nature of the geometry and the non-uniform slice 
planes within lattice itself. The Maxwell criterion is primarily driven by the relative density and assumes that the material is distributed symmetrically within the lattice structure.

From Figure 10, it is evident that Maxwell criteria underestimates the Young's modulus of designs with a relative density $<0.34$. on the other hand, when the designs featured a relative density $>0.34$, the elastic performance was overestimated. In terms of numerical accuracy, the worst agreement was for OS5 followed by IS 2 at a difference of 6.30 and $5.87 \mathrm{GPa}$ respectively. The best agreement was exhibited by IOS 1 followed by OS2 with a difference of 0.02 and $0.63 \mathrm{GPa}$ respectively.

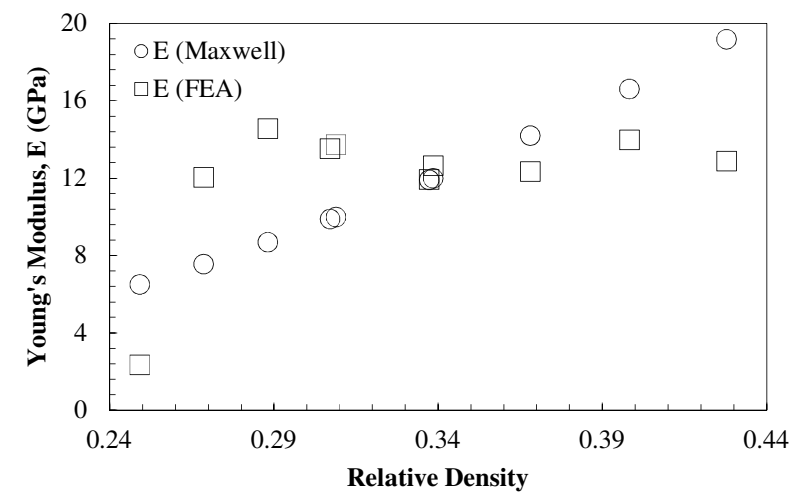

Figure 10. Influence of relative density on the prediction of Young's modulus using Maxwell criterion and FEA.

From FEA the highest stiffness was exhibited by OS5 $\left(\mathrm{E}_{\mathrm{FEA}}=19.18\right)$ which was expected due to the largest sheath thickness. The lowest was exhibited by the sheath-less design NS1. It is evident that introduction of sheathing strategy makes the mechanical behaviour of the designs complex primarily due to stress concentration.

Comparing the implant performance $\left(\mathrm{E}_{\mathrm{FEA}}\right)$ with the segmented bone $\left(\mathrm{E}_{\mathrm{bone}}=18.01 \mathrm{GPa}\right)$, designs IS2 showed the closest performance followed by OS4 at a difference of 3.44, and $4.03 \mathrm{GPa}$ respectively. However, this is primarily due to the stress concentration due to the sheathing strategy considered. Consequently, the design IOS 1 with a predicted Young's modulus of $11.94\left(\mathrm{E}_{\mathrm{FEA}}\right)$ was chosen as the best design for prototyping and experimental testing.

\subsection{Prototyping and mechanical testing}

Based on the numerical analysis the design IOS1 was additively manufactured as shown in Figure 11. As expected with DMLS, a rough surface finish was created due to the layer-by-layer melting that occurs during ALM. The implant was then tested using the compression testing machine to failure.

The stress-strain curve obtained from the experimental test shown is shown in Figure 12. The curve exhibited a typical profile that can be expected from Ti64. The test resulted in a
Young's Modulus of $14.58 \mathrm{GPa}$. Comparing the experimental Modulus with FEA, a difference of $2.66 \mathrm{GPa}$ was observed. Consequently, the FEA underestimated the Young's Modulus by $20 \%$ with respect to experimental tests.

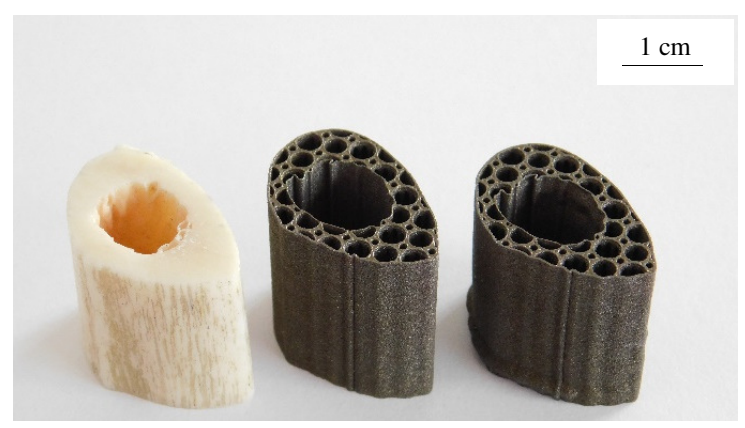

Figure 11. Additively manufactured implant prototype.

The implant exhibited an ultimate bearing strength of 1.02 $\mathrm{GPa}$, compared to the Ti64 data sheet value of $1.74 \mathrm{GPa}$. This is a percentage reduction of $58.6 \%$ at a relative density of $33.9 \%$.

The usual working stress that can be expected at the segmented tibial section based on a load of $100 \mathrm{~kg}$ for static and dynamic loading is $\sim 5.5$ and $\sim 55.5 \mathrm{MPa}$ respectively. In comparison, the implant being considered is far superior exhibiting a bearing strength approximately twice that of the dynamic working stress expected at the tibial section. However, an implant performance close to the working stress may be beneficial to accelerate the process of ossification. In comparison, the implant design IOS 1 is superior exhibiting a bearing strength of approximately $1.02 \mathrm{GPa}$.

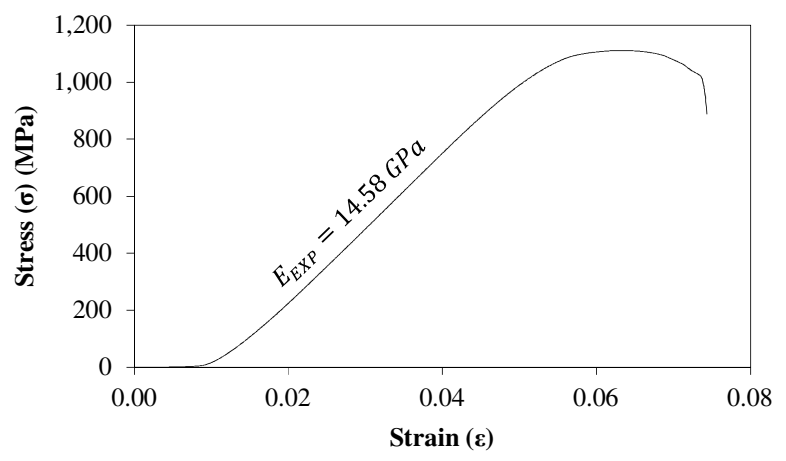

Figure 12. Experimental stress-strain curve obtained for IOS1.

\subsection{Visual inspection}

The prototype of the surface was investigated using the three-dimensional confocal microscope. The surface roughness profile was identified to be between $25 \mu \mathrm{m}$ and 90 $\mu \mathrm{m}$ with a mean value of $56.78 \mu \mathrm{m}$ as shown in Figure 13. It is evident that the semi-melted powder particles adhering to the surface was the cause of the rough surface finish. 


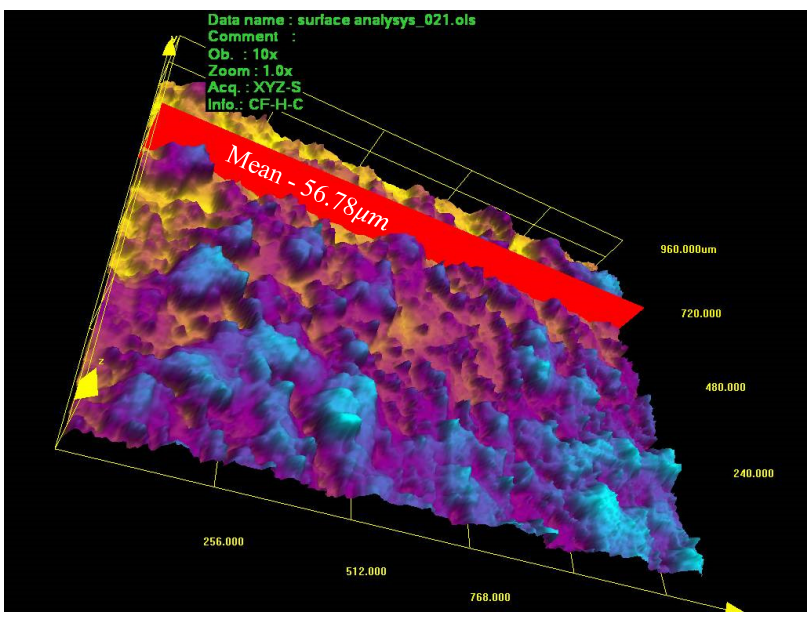

Figure 13. Surface roughness characterisation of the implant surface using confocal microscope.

\section{Discussion}

Stress shielding inhibits ossification, as such constant stress distribution through both unit cell and lattice structure was sought to facilitate bone growth. In an attempt to facilitate this, a suitable unit cell as shown in Figure 3 with a uniform stress profile (20) was chosen as the building block of the all the implant designs tested.

A total of ten implant designs with parameters as shown in Table 1 were considered under four categories as shown in Figure 4. The four categories were considered to study the influence of sheath location and thickness on performance of anatomical implant.

The performance of the implants was first characterised using the Maxwell criterion (21). This methodology uses relative density as the primary parameter to characterise the stiffness of the structure. Accordingly, the relative density $\left(\rho_{r(i m p)}\right)$ of all the ten implant designs were derived as listed in Table 3. It can be seen that the relative density of all the designs are within a 0.25 and 0.45 range which is conductive of osseointegration $(22,23)$. It must be noted that these models cannot consider the geometrical effect of sheath as Maxwell models assumes all links as beams and connections as joints.

The highest relative density of the implant was exhibited by the design OS5, which featured only an outer sheath having a thickness of $0.6 \mathrm{~mm}$. The lowest was exhibited by NS1 which featured no sheath at all.

It can also be seen that OS1 and IS3 feature similar relative densities despite the difference in sheathing strategy employed. A similar trend can be observed for designs OS2 and IOS1 despite featuring different sheathing strategies.

From Figure 6 it is evident that a higher relative density gives rise to a higher stiffness as represented by the Young's modulus. The lowest theoretical Young's modulus was exhibited by NS1 which was expected due to no sheathing strategy. The highest Young's modulus was exhibited OS5 which feature the largest outside sheath thickness.

Comparing the theoretical performance of the implant designs, OS5 and OS4 shows comparable performance with difference of $+6.29 \%$ and $-8.02 \%$ respectively with reference to the Young's modulus of the segmented tibia (18.01 GPa).

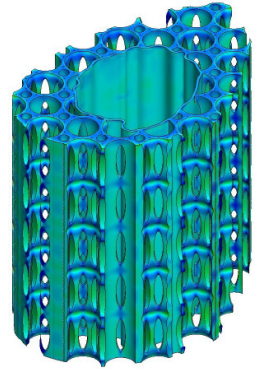

(a)

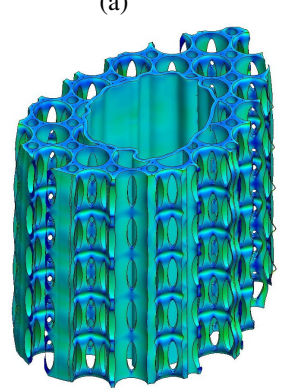

(c)

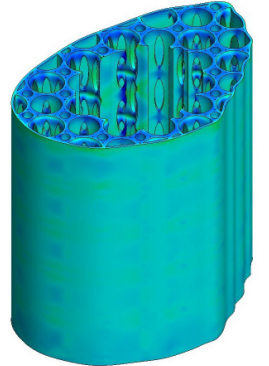

(b)

(d)

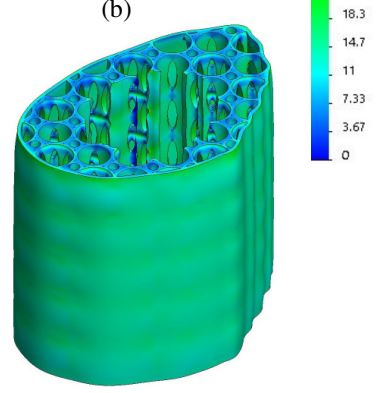

Figure 14. Stress concentration based on the sheathing strategy considered, where (a) shows the normal stress distribution (MPa) for design IS2, (b) OS1, (c) IS3 and (d) OS2.

From the finite element stress profile shown in Figure 7, it is evident that, the stress is uniformly distributed within the overall implant. However, using the external sheath would cause a higher stress concentration compared to internal sheath of similar thickness (Figure 14a-b). Furthermore, when the sheath thickness was increased to $0.3 \mathrm{~mm}$ (Figure $14 \mathrm{c}-\mathrm{d}$ ) the outer sheath is dominating as the main load bearing structure.

Comparing the normal stress for values for all the designs as shown in Figure 8. It is evident that increasing the sheath thickness induces a higher stress concentration with in the design. Increasing the inner sheath thickness from 0.1 to 0.2 , the stress concentration increased by $2.76 \%$. However, on subsequent increase in sheath thickness, the increase in stress concentration was much higher. Accordingly, to enable an even distribution of the stress, the sheath thickness must be kept to a minimum, preferably $<0.2 \mathrm{~mm}$.

For the outer sheath design, both increasing and decreasing the thickness seems to affect the stress concentration drastically. However, the stress concentration reduced at thicknesses $0.3,0.4$ and 0.5 in comparison to 0.2 and 0.6. furthermore, the outer sheath strategy seems to be 
superior from a stress perspective compared to inner sheathing and non-sheathing strategy.

Consequently, sheath thickness must be carefully chosen to not become the primary load bearing structure. The combination of inner and outer sheath ensures that the stress is uniformly distributed as shown in Figure $15 \mathrm{~b}$ compared to a non-sheathed design (Figure 15a). From this analysis, it is evident that varying the sheath location and thickness can be done to prevent or optimise for maladapted stress concentration (24).

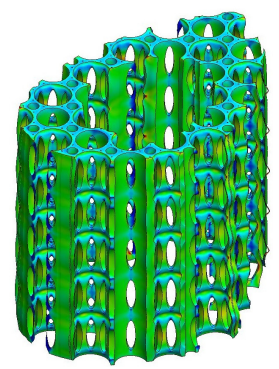

(a)

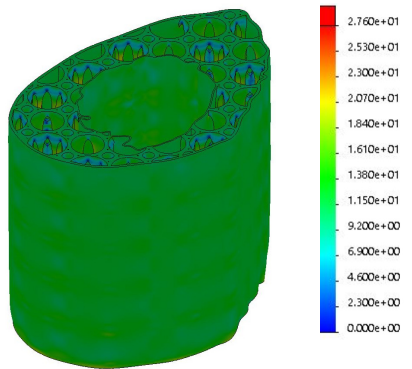

(b)
Figure 15. Stress distribution (MPa) for (a) non-sheathed design versus (b) external and internal sheath design.

In this regard, it was deemed most suitable to pursue a design incorporating both inner and outer sheath (IOS1). This design also offers additional safety due to the absence of sharp corners that is a feature in open cell anatomical designs. Furthermore, consideration was given to the DMLS manufacture process which would require unviable support structures in the case of an open pore anatomical structure.

Figure 9-10 shows that the Maxwell criteria cannot be used to predict the stiffness of sheathed anatomical implants. The Maxwell criteria underestimated the $\mathrm{E}$ at relative densities below 0.34 . and overestimated when the relative density increased. This can be due to the irregular anatomical nature of the geometry and the non-uniform lattice geometry. Furthermore, the Maxwell criterion is purely based on relative density and assumes that the material is distributed evenly within the global structure. When comparing the performance, design OS5 showed the worst agreement between the two methods at a difference of $39.3 \%$. The best agreement was exhibited by IOS 1 at a percentage difference of $0.16 \%$.

Comparing the finite element performance, NS1 and OS5 showed the lowest and highest Young's Modulus. This was expected as the design NS1 featured no sheath and OS5 the highest sheath thickness of all the designs simulated. In comparison to the Young's modulus of the segmented bone at $18.01 \mathrm{GPa}$, the best performance was exhibited by designs IS2 at a difference of $21 \%$. However, the performance of IS2 is primarily due stress concentration, consequently, IOS 1 was considered for manufacture. Based on the overall stress profile (Figure 8), the introduction of sheathing strategy makes the mechanical behaviour of the designs complex.

The numerical analysis of IOS1 resulted in a Youngs Modulus of $11.94 \mathrm{GPa}$. The FEA results were validated using the Maxwell stability criterion and experimental compression test. Comparing the simulated $\mathrm{E}$ values with experimental and analytical (Maxwell) results, a difference of $20 \%$ and $0.16 \%$ was observed respectively. When simulating lattice geometry, a difference between FEA and experimental methods of 2 folds are often reported (25-28). This can be primarily attributed to the difference in the implant geometry fabricated using SLM in comparison to the idealised CAD model. When additively manufacturing Ti64 thin wall structures below $300 \mu \mathrm{m}(<0.3 \mathrm{~mm})$, the wall is composed of fully and partially melted material layers. This effect is significantly large for IOS 1 as it features an internal and external sheath of 0.1 and $0.23 \mathrm{~mm}$ respectively.

In addition, an average layer thickness variation of 56.78 $\mu \mathrm{m}$ (Figure 13) shows there is a thickness variation of at least $\pm 0.05 \mathrm{~mm}$. Furthermore, the finite element models of the implant designs were simplified by modelling the volume representing the cellular structure as a continuum part, with a single value being assigned for the elastic modulus. Due to the nature of ALM, the properties of the sheath and unit cell are not the same. Considering this, the results of the simplified model in this study are in reasonable agreement between the two methods.

Accordingly, it can be said that the simulation procedure presented in this paper is suitable for predicting the performance of sheathed implant designs. However, it is acknowledged that attention is needed when adopting the simulation procedure for vastly different lattice architecture. It is promising to see that the Maxwell stability criterion fits well with the chosen sheathed implant considered in this study.

The experimental test curve (Figure 12) exhibited a typical profile that can be expected from Ti64. The test resulted in a Young's Modulus of $14.58 \mathrm{GPa}$ which is slightly lower than the segmented bone (18.01 GPa). The implant exhibited a bearing strength of $1.02 \mathrm{GPa}$, compared to the Ti64 bulk data of $1.74 \mathrm{GPa}$; this is a percentage reduction of $58.6 \%$ at a relative density of 0.33 .

The usual working stress that can be expected at the segmented tibial section based on a load of $100 \mathrm{~kg}$ for static and dynamic loading is $\sim 5.5$ and $\sim 55.5 \mathrm{MPa}$ respectively. In comparison, the IOS 1 is far superior exhibiting a bearing strength approximately twice that of the dynamic working stress in tibia.

Evaluating the deformation of the sample itself was outside of the scope of this study. This because in biomedical engineering, the expectation is for the implant to be in its elastic range throughout service cycle. 
The visual inspections using the confocal optical microscope revealed a mean roughness of $56.78 \mu \mathrm{m}$ (Figure 13). The results also showed that the DMLS manufacturing process delivered a consistent surface roughness across the implant topology. Considering the effect of topology on cell attachment, proliferation and extra-cellular matrix production; open porosity is needed to allow the cell to invade the structure.

From the works of Bonfield (29), particularly focusing on bone scaffolds, pore size and surface roughness of approximately $50 \mu \mathrm{m}$ can accommodate osteoblasts efficiently. Precursor osteoblasts that are of interest to this study, are around $10 \mu \mathrm{m}$, spherical with filopodia, only 0.1 $\mu \mathrm{m}$ thick, used to cling on to surfaces. However, it is important to note osteoblasts can be found in various sizes; a mature osteoblast can be over $200 \mu \mathrm{m}$. Nevertheless, the bone growth on the implant surface can be accelerated using suitable bioactive agent (2,30-32).

Taniguchi, et al., (33), compared SLM manufactured Ti lattice structures using porosity of $60 \%$, with respect to the level of bone-implant fixation and cancellous bone ingrowth, in vivo. The results showed that for ingrowth, pores around $600 \mu \mathrm{m}$ and $400 \mu \mathrm{m}$ performed best and worst respectively. In comparison, this work presents implant porosities ranging from $57-75 \%$ as listed in Table 3. Furthermore, the design featured pore sizes of 590,700 and $820 \mu \mathrm{m}$ as shown in Figure 16a-c.

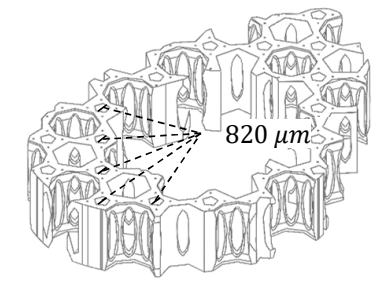

(b)

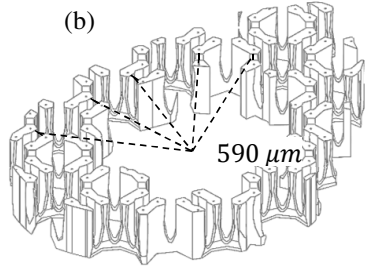

(a)

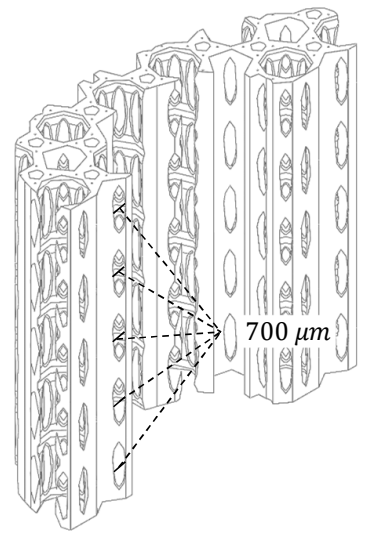

(c)
Figure 16. showing the diameter of porous cavities within the implant design (a) sectioned in top plan midway through the unit cell, (b) sectioned in top plan completion of first unit cell and (c) sectioned in right plane.

Nevertheless, it must be noted that only the top and bottom surfaces of the implant come in contact with the bone. Therefore, bone in growth is expected to occur at the top and bottom surfaces, and osteo-conduction occurs along the inner and outer sheathed surfaces when the implant is treated by suitable bioactive agent. Therefore, the only pore size that will have an influence on bone growth is the 820 $\mu \mathrm{m}$ shown in Figure 16b. The other geometrical pore sizes shown in Figure 16a and $\mathrm{c}$ has a negligible effect on bone growth.

Implant failure due to Young's Moduli mismatch between the host bone is well documented $(27,34)$. One of the most important challenges in this field is to tailor the desirable mechanical properties to minimise this failure. Titanium has strong compressive properties with relatively low Young's Modulus in comparison to steel. However; bulk titanium exceeds the Young's Modulus of cortical bone by more than three-fold resulting in stress shielding and subsequent weakening of the connection between the bone and the implant (35).

The implant design presented in this paper shows a Young's Modulus of $14.58 \mathrm{GPa}$ (experimental) which is closer to the lowest value of Young's Modulus of cortical bone (18-25 GPa). The slightly lower Young's Modulus of the presented implant in comparison with bone is beneficial to accelerate the process of osteointegration (36).

It is anticipated that after a period of bone growth, Young's Modulus of the implant will match that of surrounding bone. Overall, this will reduce the requirement for implant arthroplasty due to implant loosening (37). Furthermore, the high volumetric porosity of the implant along with rough surface roughness of Ti64 is expected to accelerate the process of osteogenesis in the presence of a suitable bioactive agent (2,30-32). Finally, the advantages of the presented implant over conventional ones includes easy implantation in surgical operation and allows for the reduction of stress shielding.

\section{Conclusion}

This research investigates the mechanical performance of Ti64 biomedical implants that anatomically mimics the tibial bone section. The design methodology to match the segmental tibial anatomy was based on data captured using X-ray tomography. A total of ten implant designs under four categories namely: no sheath (NS), inner sheath (IS), outer sheath (OS) and inner + outer sheath (IOS) were investigated. It was found that sheathing is a potential strategy worth considering to reduce stress concentration in porous implants. However, the location and thickness of the sheath must be carefully considered with respect to the surrounding porous lattice structure. For IS strategy, increasing the thickness increases the stress concentration within the implant. For external sheath, the best performance was for a thickness of $0.4 \mathrm{~mm}$, any reduction or increase was found to drastically increase the stress concentration. Overall, the best performance was observed when both an inner and outer sheathing was employed. The results of the best performing implant design (IOS1) showed a Young's Modulus of $14.08 \mathrm{GPa}$ which was lower than that of the 
tibial segmented bone. Furthermore, the measured mean surface roughness of $56.78 \mu \mathrm{m}$ is constructive for osteogenesis in the presence of a suitable bioactive agent. It is anticipated that the presented porous, sheathed implant design can be considered for both cemented and uncemented tibial segmental replacement. However, future work is required based on in-vitro incubation using bio-reactors to estimate the actual bone ingrowth within the implant under dynamic loading.

\section{Authorship Contribution Statement}

Aaron Vance: Methodology, Formal analysis, Investigation, Writing original draft. Klaudio Bari: Conceptualization, Investigation. Arun Arjunan: Validation, Investigation, Visualization, Conceptualization, Formal analysis, Writing original draft, review and editing.

\section{Acknowledgements}

This research was supported by the University of Derby, University of Wolverhampton and Renishaw plc. The bone samples used in this study were provided by Dr Graham Souch and the DMLS implants were manufactured through Alex Harris (Renishaw, UK).

\section{References}

(1) Ahmadi SM, Hedayati R, Li Y, Lietaert K, Tümer N, Fatemi A, et al. Fatigue performance of additively manufactured metabiomaterials: The effects of topology and material type. Acta Biomaterialia 2018 Jan;65:292-304.

(2) Fujibayashi S, Kim H, Neo M, Uchida M, Kokubo T, Nakamura $\mathrm{T}$. Repair of segmental long bone defect in rabbit femur using bioactive titanium cylindrical mesh cage. Biomaterials 2003;24(20):3445-3451.

(3) Karageorgiou V, Kaplan D. Porosity of 3D biomaterial scaffolds and osteogenesis. Biomaterials 2005;26(27):5474-5491.

(4) Pilliar RM. Powder metal-made orthopedic implants with porous surface for fixation by tissue ingrowth. Clinical orthopaedics and related research $1983 \mathrm{Jun}(176): 42-51$.

(5) Hulbert SF, Young FA, Mathews RS, Klawitter JJ, Talbert CD, Stelling FH. Potential of ceramic materials as permanently implantable skeletal prostheses. Journal of biomedical materials research $1970 \mathrm{Sep} ; 4(3): 433-456$.

(6) Carpenter RD, Klosterhoff BS, Torstrick FB, Foley KT, Burkus JK, Lee CSD, et al. Effect of porous orthopaedic implant material and structure on load sharing with simulated bone ingrowth: A finite element analysis comparing titanium and PEEK. Journal of the Mechanical Behavior of Biomedical Materials 2018;80:68-76.

(7) Chen Y, Frith JE, Dehghan-Manshadi A, Attar H, Kent D, Soro NDM, et al. Mechanical properties and biocompatibility of porous titanium scaffolds for bone tissue engineering. Journal of the Mechanical Behavior of Biomedical Materials 2017 Nov;75:169174.

(8) Zhang Q, Cossey A, Tong J. Stress shielding in periprosthetic bone following a total knee replacement: Effects of implant material, design and alignment. Medical Engineering \& Physics 2016;38(12):1481-1488

(9) Hutmacher DW. Scaffolds in tissue engineering bone and cartilage. Biomaterials 2000;21(24):2529-2543.

(10) Tarik Arafat M, Gibson I, Li X. State of the art and future direction of additive manufactured scaffolds-based bone tissue engineering. Rapid Prototyping Journal 2014 Jan 14,;20(1):13-26.

(11) Bari K, Osarinmwian C, López-Honorato E, Abram TJ. Characterization of the porosity in TRISO coated fuel particles and its effect on the relative thermal diffusivity. Nuclear Engineering and Design 2013;265:668-674.

(12) Rafael Ferreira Gregolin, Cecília Amelia de Carvalho Zavaglia, Ruís Camargo Tokimatsu, João A. Pereira. Biomechanical Stress and Strain Analysis of Mandibular Human Region from Computed Tomography to Custom Implant Development. Advances in Materials Science and Engineering 2017 Jan 1,;2017.

(13) Zadeh MHR, Seifi M, Abdolrahimi M, Hadavi M. A comprehensive in vitro study of the carbon nanotube enhanced chitosan scaffolds for cancellous bone regeneration. Biomedical Physics \& Engineering Express 2018 Apr 10,;4(3):35027.

(14) Basalah A, Esmaeili S, Toyserkani E. A novel additive manufacturing-based technique for developing bio-structures with conformal channels and encapsulated voids. Biomedical Physics \& Engineering Express 2015 Nov 19,;1(4):45007.

(15) Maurmann N, Pereira DP, Burguez D, de S Pereira, Frederico D A, Inforçatti Neto P, Rezende RA, et al. Mesenchymal stem cells cultivated on scaffolds formed by 3D printed PCL matrices, coated with PLGA electrospun nanofibers for use in tissue engineering. Biomedical Physics \& Engineering Express 2017 Jun 27,3(4):45005.

(16) Shadid WG, Willis A. Bone fragment segmentation from 3D CT imagery. Computerized Medical Imaging and Graphics 2018;66:14-27.

(17) Arjunan A, Wang CJ, Yahiaoui K, Mynors DJ, Morgan T, Nguyen VB, et al. Development of a 3D finite element acoustic model to predict the sound reduction index of stud based doubleleaf walls. J Sound Vibrat 2014;333(23):6140-6155.

(18) Arjunan A, Wang CJ, Yahiaoui K, Mynors DJ, Morgan T, English M. Finite element acoustic analysis of a steel stud based double-leaf wall. Build Environ 2013;67:202-210.

(19) Arjunan A, Wang C, English M, Stanford M, Lister P. A computationally-efficient numerical model to characterize the noise behavior of metal-framed walls. Metals 2015;5(3):1414-1431. 
(20) Vance A, Bari K, Arjunan A. Compressive performance of an arbitrary stiffness matched anatomical Ti64 implant manufactured using Direct Metal Laser Sintering. Materials \& Design 2018 Nov.

(21) M.F Ashby. The properties of foams and lattices. Philosophical Transactions of the Royal Society A: Mathematical, Physical and Engineering Sciences 2006 Jan 15,;364(1838):15-30.

(22) Bose S, Vahabzadeh S, Bandyopadhyay A. Bone tissue engineering using 3D printing. Materials Today 2013 Dec;16(12):496-504

(23) Wiria FE, Shyan JYM, Lim PN, Wen FGC, Yeo JF, Cao T. Printing of Titanium implant prototype. Materials and Design 2010;31:S105

(24) Eckstein F, Hudelmaier M, Putz R. The effects of exercise on human articular cartilage. Journal of Anatomy 2006 Apr;208(4):491-512.

(25) Hazlehurst KB, Wang CJ, Stanford M. An investigation into the flexural characteristics of functionally graded cobalt chrome femoral stems manufactured using selective laser melting. Materials and Design 2014 Aug;60:177-183.

(26) Harrysson OLA, Cansizoglu O, Marcellin-Little DJ, Cormier DR, West HA. Direct metal fabrication of titanium implants with tailored materials and mechanical properties using electron beam melting technology. Materials Science \& Engineering C 2008;28(3):366-373.

(27) Parthasarathy J, Starly B, Raman S. A design for the additive manufacture of functionally graded porous structures with tailored mechanical properties for biomedical applications. Journal of Manufacturing Processes 2011;13(2):160-170.

(28) Hazlehurst K, Wang CJ, Stanford M. Evaluation of the stiffness characteristics of square pore CoCrMo cellular structures manufactured using laser melting technology for potential orthopaedic applications. Materials and Design 2013 Oct;51:949955

(29) William Bonfield. Designing porous scaffolds for tissue engineering. Philosophical Transactions of the Royal Society A: Mathematical, Physical and Engineering Sciences 2006 Jan $15, ; 364(1838): 227-232$.

(30) Kim H-, Kokubo T, Fujibayashi S, Nishiguchi S, Nakamura T. Bioactive macroporous titanium surface layer on titanium substrate. J Biomed Mater Res 2000;52(3):553-557.

(31) Inui K, Maeda M, Sano A, Fujioka K, Yutani Y, Sakawa A, et al. Local application of basic fibroblast growth factor minipellet induces the healing of segmental bony defects in rabbits. Calcif Tissue Int 1998;63(6):490-495.

(32) Fujibayashi S, Nakamura T, Nishiguchi S, Tamura J, Uchida $\mathrm{M}$, Kim H-, et al. Bioactive titanium: Effect of sodium removal on the bone-bonding ability of bioactive titanium prepared by alkali and heat treatment. J Biomed Mater Res 2001;56(4):562-570.
(33) Taniguchi N, Fujibayashi S, Takemoto M, Sasaki K, Otsuki B, Nakamura T, et al. Effect of pore size on bone ingrowth into porous titanium implants fabricated by additive manufacturing: An in vivo experiment. Materials Science \& Engineering C 2016 Feb $1, ; 59: 690-701$

(34) Heary RF, Parvathreddy N, Sampath S, Agarwal N. Elastic modulus in the selection of interbody implants. Journal of spine surgery (Hong Kong) 2017 Jun;3(2):163.

(35) Pałka K, Pokrowiecki R. Porous Titanium Implants: A Review. Advanced Engineering Materials 2018 May;20(5):n/a.

(36) Simon U, Augat P, Ignatius A, Claes L. Influence of the stiffness of bone defect implants on the mechanical conditions at the interface - a finite element analysis with contact. Journal of Biomechanics 2003;36(8):1079-1086.

(37) Dattani R. Femoral osteolysis following total hip replacement. Postgraduate medical journal 2007 May;83(979):312-316. 